\title{
Influencia de un tercer juego de cuchillas en la eficiencia del proceso de extracción de la sacarosa de la caña de azúcar
}

\author{
Influence of a Third Set of Knives on the Efficiency of the Sucrose \\ Extraction from Sugar Cane
}

\author{
Corrales-Suárez Jorge Michel \\ Universidad de Las Tunas \\ Facultad de Ciencias Técnicas \\ Centro de Estudio de Energía y Procesos Tecnológicos \\ Correo:jorgecs@ult.edu.cu \\ Gil-Ortiz José Marcos \\ Universidad de Las Tunas \\ Facultad de Ciencias Técnicas \\ Centro de Estudio de Energía y Procesos Tecnológicos \\ Correo:jgil@ult.edu.cu \\ Remédios-Castañeiras Pedro Dionisio \\ Universidad de Las Tunas \\ Facultad de Ciencias Técnicas \\ Centro de Estudio de Energía y Procesos Tecnológicos \\ Correo:pdremedio@ult.edu.cu
}

\author{
Masjuan-Leyva Yurisleydis \\ Empresa Logística Agropecuaria \\ Dirección de Operaciones \\ Correo:yml@elalt.co.cu \\ Gil-Ceballo José Alexander \\ Empresa Nacional de Desarrollo de Software Las Tunas \\ Departamento de programación \\ Correo: jgilc@lt.desoft.cu
}

Información del artículo: recibido: noviembre de 2014, reevaluado: marzo de 2015, aceptado: abril de 2015

\section{Resumen}

Una de las variables que influye sobre la eficiencia en el proceso de extracción de la sacarosa de la caña de azúcar en tándem de molinos, es el índice de preparación de la caña. Esta influencia se puede medir a través de \% de sacarosa $(\% \mathrm{Pol})$ y $\%$ de Humedad en el bagazo final y la demanda de potencia del tándem, estas tres variables constituyen las variables dependientes de esta investigación. La variable independiente es el índice de preparación de la caña. El objetivo de esta investigación es mostrar el impacto de un aumento del índice de preparación de la caña, logrado con el aumento de un tercer juego de cuchillas en el tándem, en la eficiencia del proceso de extracción y la demanda de potencia del tándem. La investigación se realizó en dos etapas. Una primera etapa donde se operó el tándem con los dos juegos de cuchillas existentes y una segunda etapa donde se operó con tres juegos de cuchillas, en donde se montó el tercer juego de cuchillas para incrementar el índice de preparación de la caña. Mediante un análisis estadístico se concluye que al aumentar el índice de preparación de la caña los \% Pol y \% Humedad del bagazo final disminuyen significativamente y la demanda de potencia del tándem disminuye $2.87 \%$.

\section{Descriptores:}

- preparación de la caña de azúcar

- pol

- humedad

- potencia

- tándem 


\begin{abstract}
The index of cane preparation is one of the variables that influence on efficiency the sucrose extraction process from sugar cane mills in tandem. This influence can be measured by the \% sucrose (\% Pol) and \% humidity in the final bagasse and power demand tandem, these three variables are the dependent variables on this research. The index of cane preparation was the independent variable. The objective of this research is to show the impact of an increased rate of cane preparation, achieved with addition of a third set of knives in tandem, on the efficiency of the extraction process and the tandem power demand. This research was carried out in two stages. A first stage where the tandem was operated with two sets of existing knives and a second stage where it was operated with the addition of a set of knives (that to say with three sets of knives), the third set of knives mounted to increase the rate of cane preparation. A statistical analysis concludes that with increasing the rate of cane preparation both in the final bagasse the \% Pol and \% Humidity decrease significantly and tandem power demand decreases by $2.87 \%$.
\end{abstract}

\section{Introducción}

El proceso industrial de producción de azúcar de caña, está compuesto por un primer proceso que consiste en la preparación de la caña y posterior extracción de la sacarosa (\% Pol) que esta contiene. La preparación de la caña se realiza mediante equipos especialmente diseñados para este fin, como la desmenuzadora, la desfibradora y las cuchillas giratorias (Hugot, 1986; Jenkins, 1971). El costo de la energía que consume una central azucarera es tan bajo (producto que se autoabastece energéticamente), que aunque en el área de preparación de la caña se requiera un considerable aumento del consumo de energía para incrementar el índice de preparación de la caña, el costo de esa energía se compensa con cualquier ganancia en el proceso de extracción por pequeña que sea, o con la disminución de la demanda de potencia en el área de molienda (Jenkins, 1971). Al determinar el índice de preparación de la caña (Morejón y Revé, 2013), un buen índice de ella favorece extraordinariamente el trabajo de la planta moledora, atrayendo cuantiosos beneficios económicos, pues se obtiene mayor capacidad de molida, mayor extracción en el tándem y menor demanda de potencia en los molinos (Jenkins, 1971).

Se han publicado artículos donde se investiga el proceso tecnológico para incrementar el índice de preparación de la caña de azúcar con el objetivo de evaluar su eficiencia y detectar los principales problemas en dicho proceso (Morejón y Revé, 2013), pero solo se limitan al proceso de preparación de la caña. Otros autores han estudiado la influencia de la preparación de la caña sobre la compresibilidad del bagazo a escala de laboratorio (Gil, 2011; Gil et al., 1998).

Inskip (2010) demuestra que al mejorar el índice de preparación de la caña, no importa del modo que se haga, los beneficios son alentadores, porque se propicia una disminución de la demanda de potencia de la planta moledora.

Se ha demostrado a nivel de laboratorio que al tratar la caña de azúcar con microondas las fuerzas de interacción entre las células se reduce a $20 \%$ de su valor sin tratar. Esta reducción hace que sea más fácil de comprimir la caña y conduce a un aumento considerable de la extracción del jugo de la caña, logrando una disminución en el \% Pol (Brodie y Harris, 2011).

El jugo se extrae con mayor facilidad de un material finamente dividido, ya que al no tener que consumir energía en romper la estructura de la caña la mayor parte de la energía suministrada al molino se emplea en extraer el jugo del colchón de bagazo, lo que disminuye la potencia requerida por la molienda (Jenkins, 1971). El índice de preparación de la caña está relacionado con la proporción de células abiertas. Entre mayor sea este, más acceso tendrá el agua de imbibición al jugo, que en otro caso, solo se diluiría por difusión a través de las paredes y las membranas celulares.

Una preparación de la caña que produzca fibras delgadas y largas es más conveniente que la que dé un polvillo fino, pues la primera produce partículas que pueden entrelazarse entre sí creando una especie de agarre entre ellas, lo que facilita la toma del material por el molino, en cambio, la segunda dificulta la alimentación. El tamaño de partícula con que se alimenta el molino no debe ser mayor de $40 \mathrm{~cm}$; aunque se considera que entre 15 y $25 \mathrm{~cm}$ es el más satisfactorio en la operación (Hamill, 1972). Corrales et al. (2013) demuestra que el \% de sacarosa (\% Pol) en bagazo final depende fundamentalmente de la preparación de la caña.

Loughran y Kannapiran (2002) desarrollaron un modelo computacional para estudiar la influencia de la 
preparación de la caña en el proceso de extracción, con el objetivo de mejorar la extracción de la sacarosa y minimizar las humedades finales, en ese modelo tienen en cuenta la influencia de la velocidad de drenaje del jugo a través de los poros del bagazo, sin embargo no estudian la inclusión de un tercer juego de cuchillas para mejorar el índice de preparación de la caña.

Jenkins (1971) señala que el índice de preparación de la caña influye sobre las pérdidas por reabsorción en bagazo. Estas pérdidas van disminuyendo con el aumento de la preparación hasta un grado determinado a partir del cual la relación de reabsorción a extracción comienza a aumentar, por lo que se plantea que existe un óptimo para el cual son mínimas estas pérdidas. En la literatura consultada no existe referencia de estudios sobre la influencia de un tercer juego de cuchillas en el proceso de extracción de la sacarosa de la caña de azúcar.

La investigación tuvo como objetivo demostrar que es posible mejorar el proceso de extracción de la sacarosa de la caña de azúcar con el aumento del índice de preparación de la caña mediante la introducción de un tercer juego de cuchillas, y disminuir la demanda de potencia en el tándem.

\section{Materiales y métodos}

El tándem de molinos tiene una capacidad de molida de 9200 t/día (800 000 @/día), está compuesto por seis molinos de tres mazas con cuarta maza o maza alimentadora y tiene tolva alimentadora Donnelly en todos los molinos. Se analizan como variables dependientes \% Pol, \% Humedad y demanda de potencia del tándem, como variable independiente el índice de preparación de la caña. Los valores de todas las variables se tomaron de los reportes diarios del Laboratorio Azucarero y la demanda de potencia del tándem por medio de un analizador de redes instalado en la planta eléctrica.

Los análisis se realizaron en dos etapas, una primera etapa donde se operó el tándem con dos juegos de cuchillas y una segunda etapa donde se operó con tres juegos de cuchillas. El método estadístico utilizado en esta investigación fue una comparación de dos muestras mediante prueba de hipótesis, con el objetivo de identificar si existe diferencia significativa entre las variables dependientes al trabajar con diferentes índices de preparación de la caña, el estadístico utilizado es la t de Student.

Los primeros 32 días de la zafra se preparó la caña con dos juegos de cuchillas hasta que se logró poner en marcha el tercer juego de cuchilla. Después de la instalación de este se operó durante 58 días. Los últimos 24 días de la zafra se trabajó nuevamente con dos juegos de cuchillas, ya que por problemas mecánicos quedó fuera de servicio el que se había instalado.

\section{Resultados y discusión}

En la investigación, la hipótesis nula se plantea como la igualdad entre las medias (media2 (con dos juegos de cuchillas) $=$ media 3 (con tres juegos de cuchillas)) contra una hipótesis alterna que refiere la desigualdad entre las medias (media2 $<>$ media3).

El índice de preparación de la caña promedio con dos juegos de cuchillas fue de $60.11 \%$ y con tres juegos de cuchillas $74.03 \%$.

\section{Análisis estadístico}

Para analizar si existe diferencia significativa entre las variables dependientes (\% Pol, \% Humedad y demanda de potencia del tándem) al trabajar con diferentes índices de preparación de la caña se tomó el nivel de significación $\alpha=0.05$.

En la zafra se molió con dos juegos de cuchillas durante 56 días (los primeros 32 y los últimos 24) y 58 días con tres juegos de cuchillas, todos los valores de sesgo estandarizado y curtosis estandarizado se encuentran en el rango de -2 a 2 para cada variable dependiente (tabla 1), lo que indica que los valores de las variables dependientes siguieron una distribución normal.

Tabla 1. Valores de los parámetros estadísticos obtenidos en la comparación de las muestras

\begin{tabular}{lcccccc}
\hline & \multicolumn{2}{c}{ Pol (\%) } & \multicolumn{2}{c}{ Humedad (\%) } & \multicolumn{2}{c}{ Demanda de potencia (MW) } \\
\hline & $\begin{array}{c}\text { Dos juegos de } \\
\text { cuchillas }\end{array}$ & $\begin{array}{c}\text { Tres juegos de } \\
\text { cuchillas }\end{array}$ & $\begin{array}{c}\text { Dos juegos de } \\
\text { cuchillas }\end{array}$ & $\begin{array}{c}\text { Tres juegos de } \\
\text { cuchillas }\end{array}$ & $\begin{array}{c}\text { Dos juegos de } \\
\text { cuchillas }\end{array}$ & $\begin{array}{c}\text { Tres juegos } \\
\text { de cuchillas }\end{array}$ \\
\hline Recuento & 56 & 58 & 56 & 58 & 56 & 58 \\
Promedio & 2.33911 & 2.25586 & 51.1305 & 50.3355 & 2.95143 & 2.86983 \\
Desviación estándar & 0.125282 & 0.100822 & 0.900746 & 0.640921 & 0.0748921 & 0.0715123 \\
Mínimo & 2.14 & 2.06 & 49.7 & 49.27 & 2.81 & 2.71 \\
Máximo & 2.66 & 2.45 & 52.9 & 51.8 & 3.08 & 3.01 \\
Sesgo estandarizado & 1.9272 & 0.347763 & 0.390904 & 1.61198 & -0.348899 & 1.56079 \\
Curtosis estandarizada & -0.175252 & -1.37288 & -1.85087 & -0.822825 & -1.81076 & -0.90434 \\
\hline
\end{tabular}




\section{Efecto de la variación del índice de preparación de la caña sobre el \% Pol en bagazo final}

Para la preparación de la caña con dos juegos de cuchillas los valores de los \% Pol en bagazo se encontraron entre $2.14 \%$ a $3.06 \%$ (figura 1). La inclusión de un tercer juego de cuchillas provocó un considerable aumento del índice de preparación de la caña, influyendo en una disminución de los \% Pol a valores dentro de un rango de $2.06 \%$ a $2.45 \%$ (figura 1). En los últimos 24 días de zafra el índice de preparación disminuyó nuevamente, coincidiendo con un aumento de los \% Pol en bagazo a valores dentro del rango de los primeros días de zafra (figura 1). Se observa que para los últimos días de molienda con los tres juegos de cuchillas la tendencia de los \% Pol fue de un ligero aumento, esto estuvo dado por problemas de operación de los molinos. El intervalo de confianza para la diferencia entre las medias se extiende desde 0.0411157 hasta 0.125374 . De acuerdo con que el intervalo no contiene el valor cero, existe diferencia estadísticamente significativa entre las medias de las dos muestras para un nivel de confianza de $95 \%$. Este resultado indica que el \% Pol en bagazo final depende fuertemente de la preparación de la caña.

\section{Efecto de la variación del índice de preparación de la caña sobre el \% Humedad en bagazo final}

Para la preparación de la caña con dos juegos de cuchillas las humedades se comportaron dentro de un rango de $49.70 \%$ a $52.90 \%$ (figura 2). La inclusión de un tercer juego de cuchillas dio valores de humedad dentro de

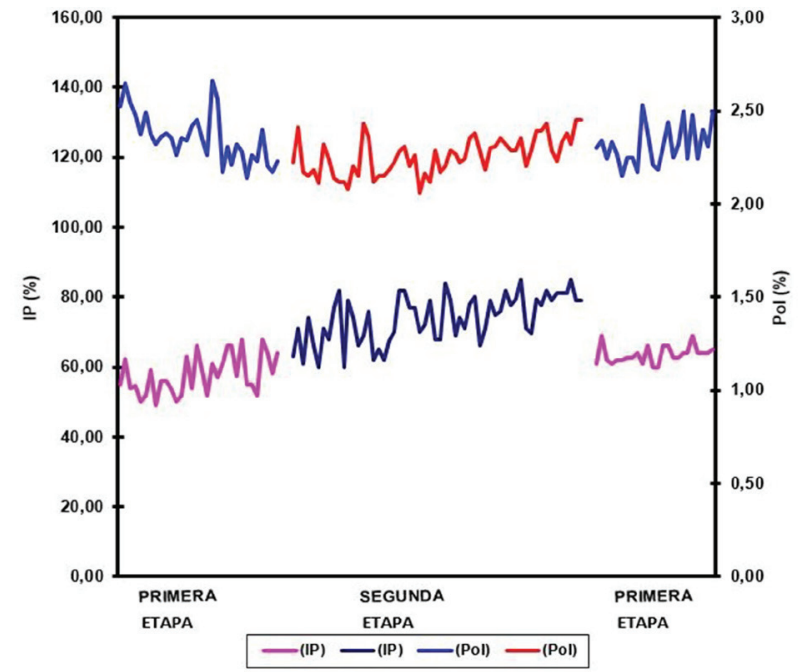

Figura 1. Comportamiento del índice de preparación de la caña sobre el \% Pol en bagazo final un rango de $49.27 \%$ a $52.40 \%$ (figura 2). En los últimos 24 días de zafra el índice de preparación disminuyó y las humedades aumentaron ligeramente hasta valores dentro del mismo rango de los primeros días de zafra (figura 2). El intervalo de confianza para la diferencia entre las medias se extiende desde 0.505698 hasta 1.08434. Puesto que este intervalo no contiene el valor cero, existe diferencia estadísticamente significativa entre las medias de las dos muestras para un nivel de confianza de $95 \%$. Estos resultados muestran que la humedad del bagazo final tiene dependencia del índice de preparación de la caña.

\section{Efecto de la variación del índice de preparación de la caña sobre la potencia demanda del tándem de molinos}

Preparando la caña con dos juegos de cuchillas la demanda de potencia del tándem osciló entre 2.81MW y 3.08MW (figura 3), rango ligeramente mayor que cuando se preparó con tres juegos de cuchillas que varió desde 2.78MW hasta 3.03MW (figura 3), en los últimos 24 días de zafra la demanda de potencia tuvo un ligero aumento hasta comportarse dentro del rango de los primeros días de zafra (figura 3). De interés particular es el intervalo de confianza para la diferencia entre las medias, el cual se extiende desde 0.0544321 hasta 0.10877 . Puesto que el intervalo no contiene el valor cero, existe diferencia estadísticamente significativa entre las medias de las dos muestras, con un nivel de confianza de $95 \%$. Estos resultados demuestran que mientras mejor es el índice de preparación de la caña menor es el con-

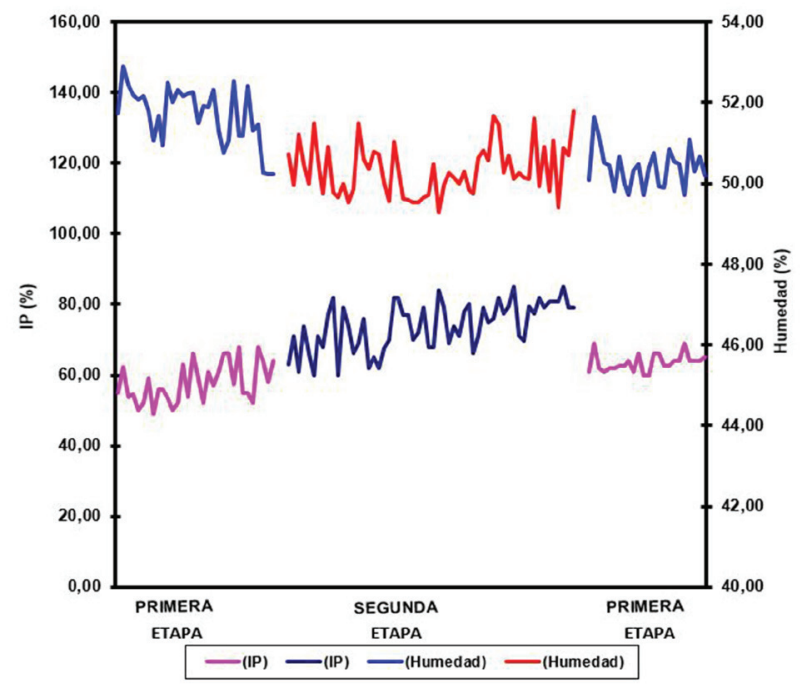

Figura 2. Comportamiento del índice de preparación de la caña sobre la humedad del bagazo final 


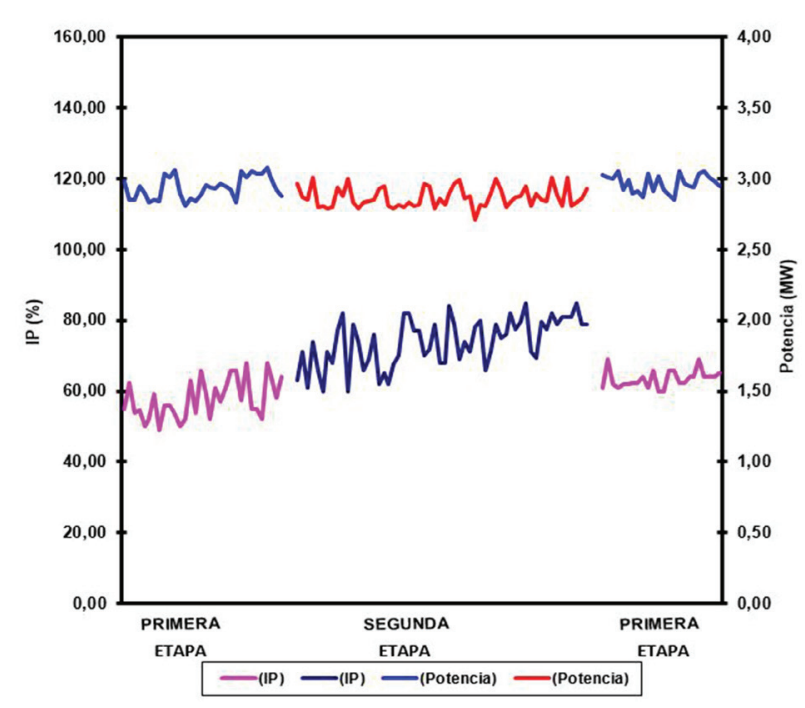

Figura 3. Comportamiento del índice de preparación de la caña en la potencia demandada del tándem

sumo de energía en los molinos del tándem para la extracción (figura 3).

Como resultado se obtiene que para las variables dependientes existe diferencia significativa entre las medias al moler con dos y tres juegos de cuchillas, respectivamente. Si esto es así entonces se puede señalar que disminuyó la reabsorción, corroborando lo que plantea Jenkins (1971).

\section{Ahorro de energía del tándem}

Estableciendo una comparación entre la potencia promedio demandada del tándem con la preparación de la caña mediante dos y tres juegos de cuchillas se obtiene una disminución de $2.71 \%$ (tabla 2).

Tabla 2. Potencia demandada del tándem para los diferentes índices de preparación de la caña

\begin{tabular}{rc}
\hline Preparación de la caña & Potencia demandada $(M W)$ \\
\hline Con dos juegos de cuchillas & 2.95 \\
Con tres juegos de cuchillas & 2.87 \\
\hline
\end{tabular}

Para la zafra analizada se molió con dos juegos de cuchillas durante 56 días, para este tiempo el ahorro de energía en el tándem se puede calcular mediante la ecuación (1), teniendo en cuenta que el tándem de molinos está compuesto por seis molinos, el índice i corre desde uno hasta seis.

$A e=\left(\sum_{i=1}^{6} N_{d o s, i}-\sum_{i=1}^{6} N_{\text {tres }, i}\right) * 56$ día $* 24 \frac{h}{d i ́ a}$
Donde:

Ae = ahorro de energía (MWh)

$\sum_{i=1}^{6} N_{d o s}=$ potencia demandada por el tándem con la preparación de la caña con dos juegos de cuchillas (MW)

$\sum_{i=1}^{6} N_{\text {tres }}=$ potencia demandada por el tándem con la preparación de la caña con tres juegos de cuchillas (MW)

El ahorro de energía en el tándem durante los días que se preparó la caña con tres juegos de cuchilla fue de 107.52 MWh.

\section{Conclusiones}

1. Al aumentar la preparación de la caña aumenta la extracción de la sacarosa de la caña de azúcar y disminuye la humedad en el bagazo final.

2. Al aumentar la preparación de la caña, es menor la demanda de potencia del tándem, lo que implica un ahorro de energía en el área de molienda.

\section{Referencias}

Brodie G., Harris G. Microwave modification of sugar cane to enhance juice extraction during milling. Revista Microwave Power and Electromagnetic Energy [en línea], volumen 45 (número 4), octubre-diciembre de 2011 [fecha de consulta: 20 de Junio de 2014]. Disponible en: http://jmpee.org/jmpee_site/Vol_45\% 284\%29/JMPEE45-4-178-Graham.pdf. ISSN: 0832-7823.

Corrales J.M., Gil J.M., Remédios P.D., et al. Ahorro energético en tándem de molinos de caña de azúcar mediante regulación de presiones hidráulicas. Revista Ingeniería Mecánica [en línea], volumen 16 (número 3), septiembre-diciembre de 2013 [fecha de consulta: 20 de junio de 2014]. Disponible en: www.ingenieriamecanica.cujae.edu.cu/index.php/revistaim/article/ download/463/819. ISSN 1815-5944.

Gil J.M. Intensificación del proceso de extracción de la sacarosa de la caña de azúcar con el uso de surfactantes aniónicos en el agua de imbibición [en línea] 2011 [fecha de consulta: 22 de junio de 2014]. Disponible en: http://www.eumed.net/tesis/2011/jmgo/index.htm. ISBN-13: 978-84-15547-35-8.

Gil J.M., Gil J.A., Suárez W. Estudio de la compresibilidad del bagazo a escala de Laboratorio. Revista Tecnológica Química [en línea] volumen 18 (número 1), enero-mayo 1998 [fecha de consulta: 22 de junio de 2014]. Disponible en: http://ojs. uo.edu.cu/index.php/tq/article/view/1606/1182. ISSN 22246185.

Hamill T.M. Wet milling extraction process and apparatus therefor. Patente 36695931 United States Patent Office, 1972 [fecha 
de consulta: 18 de julio de 2014]. Disponible en: http://www. google.com/patents/US3695931

Hugot E. Handbook of cane sugar engineering, Amsterdam, Editorial Elsevier, 1986.

Inskip S.T. Cane preparation. Optimised technology. Proc Int Soc Sugar Cane Technol [en línea] volumen 27, 2010 [fecha de consulta: 20 de julio de 2014]. Disponible en: http://pdf.thepdfportal.com/PDFFiles/10693.pdf

Jenkins G. Introducción a la tecnología del azúcar de caña, La Habana, Ed. Ciencia y Técnica, 1971.

Loughran J.G., Kannapiran A. Finite element modelling of the crushing of prepared cane and bagasse. Proceedings of the Australian Society of Sugar Cane Technologists, volumen 4, 2002 [fecha de consulta: 15 de agosto de 2014]. Disponible en: http://eprints.qut.edu.au/12510/1/12510.pdf

Morejón M.Y., Revé M.J. Influencia de la preparación de caña de azúcar a moler en la producción de azúcar en el Complejo Agroindustrial Azucarero Manuel Fajardo. Revista Ciencias Técnicas Agropecuarias, volumen 22 (número 1), enero-marzo 2013 [fecha de consulta: 28 de septiembre de 2014]. Disponible en: http://www.rcta.unah.edu.cu/index.php/rcta/article/download/186/pdf. ISSN: 1010-2760.

\section{Este artículo se cita:}

\section{Citación estilo Chicago}

Corrales-Suárez, Jorge Michel, Jose Marcos Gil-Ortiz, Pedro Dionisio Remedios-Castaneiras, Yurisleydis Masjuan-Leyva, José Alexander Gil-Ceballo. Influencia de un tercer juego de cuchillas en la eficiencia del proceso de extracción de la sacarosa de la caña de azúcar. Ingeniería Investigación y Tecnología, XVI, 04 (2015): 599-604.

\section{Citación estilo ISO 690}

Corrales-Suárez J.M., Gil-Ortiz J.M., Remedios-Castaneiras P.D., Masjuan-Leyva Y., Gil-Ceballo J.A. Influencia de un tercer juego de cuchillas en la eficiencia del proceso de extracción de la sacarosa de la caña de azúcar. Ingeniería Investigación y Tecnología, volumen XVI (número 4), octubre-diciembre 2015: 599-604.

\section{Semblanzas de los autores}

Jorge Michel Corrales-Suárez. Ingeniero mecánico graduado en la Universidad de Camagüey, Cuba. Actualmente trabaja como profesor e investigador en el Centro de Estudio de Energía y Procesos Tecnológicos de la Universidad de Las Tunas, Cuba. Es profesor de termodinámica y mecánica de los fluidos-máquinas de flujo. Sus líneas de investigación son: la eficiencia energética y los procesos tecnológicos. Actualmente es candidato a doctor en ciencias técnicas.

José Marcos Gil-Ortiz. Licenciado en física, ingeniero químico, master en ingeniería química y doctor en ciencias técnicas. Actualmente trabaja como profesor e investigador en el Centro de Estudio de Energía y Procesos Tecnológicos de la Universidad de Las Tunas, Cuba. Es profesor de operaciones unitarias de los procesos químicos. Sus líneas de investigación son: proceso de extracción en tándem de molinos cañeros y eficiencia energética de procesos.

Pedro Dionisio Remédios-Castañeiras. Ingeniero mecánico, master en ingeniería química y doctor en ciencias técnicas. Actualmente trabaja como profesor e investigador en el Centro de Estudio de Energía y Procesos Tecnológicos de la Universidad de Las Tunas, Cuba. Es profesor de operaciones unitarias de los procesos químicos. Sus líneas de investigación son: mecánica de los fluidos y sistemas de bombeo, flujos viscosos en la industria azucarera y eficiencia energética de procesos.

Yurisleydis Masjuan-Leyva. Ingeniera industrial graduada en la Universidad de Las Tunas. Cuba. Actualmente se desempeña cono especialista energética en el Departamento de Ingeniería y Desarrollo de la Empresa Logística Agropecuaria Las Tunas.

José Alexander Gil-Ceballo. Es ingeniero químico y máster en informática aplicada. Es colaborador del Centro de Estudio de Energía y Procesos Tecnológicos de la Universidad de Las Tunas, Cuba. Su línea de investigación es: gestores de negocios. 